\title{
Randomized Comparison of Thrombus Resolution Outcome between Direct Xa Inhibitor Anticoagulant and Warfarin In Acute Deep Vein Thrombosis
}

\author{
Jae Hoon Lee, Ki Hyuk Park \\ Division of Vascular Surgery, Department of Surgery, Daegu Catholic University School of Medicine, Daegu, Korea
}

Received July 24, 2020

Revised September 13, 2020

Accepted September 15, 2020

\section{Correspondence to:}

Ki Hyuk Park

Division of Vascular Surgery,

Department of Surgery, Daegu

Catholic University School of

Medicine, $17-33 \mathrm{St}$.

Dooryou-Gongwon, Nam-gu,

Daegu 42472, Korea

Tel: $+82-53-650-4065$

Fax: +82-53-624-7185

E-mail: khpark@cu.ac.kr

\begin{abstract}
Purpose: Newly developed factor Xa inhibitor anticoagulants (Xaban) produced non-inferior outcomes in terms of venous thromboembolism (VTE) recurrence and safety. On the other hand, thrombus resolution (TR), a factor associated with VTE recurrence and post-thrombotic syndrome, was not expressed clearly in these studies. This study compared the long-term TR outcomes in patients treated with Xaban or warfarin.

Methods: Fifty-seven acute deep vein thrombosis (DVT) patients were enrolled. After the initial heparin therapy, randomly assigned patients received oral Xaban or warfarin. The time in the therapeutic range was maintained at more than $60 \%$ during the anticoagulation period. After 3-12 months on the medication, a duplex scan was performed to diagnose the thrombus changes.

Results: Unprovoked DVT occurred in $80.7 \%$ of patients $(46 / 57)$, and the mean age was $60.1 \pm 13.99$ years; 26 patients were female. Concurrent pulmonary embolism was diagnosed in 20 patients (52.6\%) in the Xaban group and 10 (52.6\%) in the warfarin group. The complete TR rate for the proximal venous segment was $47.4 \%$ (18/38) for the Xaban group and $57.9 \%(11 / 19)$ for the warfarin group. Complete DVT TR was observed in $26.3 \%$ of patients $(10 / 38)$ in the Xaban group and $42.1 \%(8 / 19)$ in the warfarin group. No significant difference was observed between the proximal $(\mathrm{P}=0.657)$ and complete $\mathrm{TR}$ rates $(\mathrm{P}=$ 0.227 ) between the two groups.

Conclusion: Xaban administration for acute DVT showed no difference in TR compared to warfarin. Xaban could also be an easy-to-use anticoagulation treatment option because it does not require monitoring and has a short half-life.
\end{abstract}

Keywords: Deep vein thrombosis, Anticoagulants, Warfarin

\section{INTRODUCTION}

Deep vein thrombosis (DVT) is a significant healthcare concern resulting in serious morbidity, mortality and resource expenditure. Appropriate anticoagulation therapy is crucial for preventing early and late episodes of recurrent venous thromboembolism (VTE). However, the cumulative recurrence rate after cessation is reported as $11.0 \%, 29.1 \%$ and $39.9 \%$ after 1,5 and 10 years, respectively.(1) In a systematic review, the rate of VTE recurrence after 24 months was 3.3\% per patient year for all patients with transient risk factor and $7.4 \%$ per patient year after unprovoked VTE.(2) The persistence of residual vein thrombosis (RT) is also considered an important risk factor for recurrence. Prandoni et al.(3) reported that RT has a hazard ratio for recurrence of 2.4 (confidence interval (CI): 1.3-4.4) and 
Young et al.(4) reported a hazard ratio of 2.2 (CI: 1.19-4.21). RT also has an important late clinical consequence in VTE, which is post-thrombotic syndrome (PTS). In our cohort of patients with DVT, who were followed for two years, moderate or severe PTS signs were detected in $10 \%$ and $3 \%$, respectively, and these results were closely associated with the persistence of vein obstruction, which is the main cause of chronic venous hypertension.(5)

However, thrombus resolution (TR) and vein patency recovery are unpredictable during anticoagulation. Several studies evaluated TR with ultrasonography in symptomatic DVT patients and normalization rates at 1 year ranged from 36\%-96\% with conventional anticoagulation. $(6,7)$ Caprini et al.(8) reported that, during warfarin therapy, the international normalized ratio (INR) values were significantly higher in patients with complete DVT resolution. Recently developed new oral anticoagulants (NOACs), such as factor Xa inhibitor anticoagulants (Xaban), have advantages that make them a beneficial treatment option with consistent anticoagulation effects.(9-11) However, recanalization of thrombosed venous segments and evolution of thrombus were not clearly expressed in these studies. We performed a prospective randomized controlled study to compare the degree and rate of TR between warfarin and Xaban therapy.

\section{METHODS}

Fifty-seven consecutive patients (26 women, 31 men) with acute leg DVT were enrolled in this randomized, double-blind study after providing informed consent. The study uses a design in which all patients receive either active Xaban and warfarin placebo, or active warfarin and Xaban placebo, along with regular blood testing with reporting of real or sham INR results. Randomization was performed using of an interactive, Web-based system. All patients were hospitalized for proper diagnosis and treatment.

A duplex scan was done for DVT extent and chest CT scan used for diagnosis of pulmonary embolism for initial diagnosis. The protein C, S level and factor V anomaly as hypercoagulability test were checked before anticoagulation. Initial treatment was started with low molecular heparin for at least 5 days.

Either Xaban or warfarin was administered in a double-blind, double-dummy fashion. Xaban was started after discontinuation of initial heparin with recommended dose for patient condition. The Xaban regimen is $60 \mathrm{mg}$ orally once daily, taken with or without food. A reduced dose of Xaban of $30 \mathrm{mg}$ once daily is used for patients with moderate renal impairment (creatinine clearance of 30-50 $\mathrm{mL} / \mathrm{min}$ ), body weight of $\leq 60 \mathrm{~kg}$, or who are receiving strong P-glycoprotein inhibitors. Xaban is started after discontinuing initial heparin, either $12 \pm 3 \mathrm{~h}$ after the last dose of the twice-daily Xaban regimen, $24 \pm 3$ h after the last dose of the once-daily Xaban regimen, or $4 \pm 1 \mathrm{~h}$ after discontinuing intravenous unfractionated heparin.

Warfarin was started concurrently with the study regimen of heparin with adjustment of the dose to maintain the international normalized ratio (INR) between 2.0 and 3.0. All measurements were performed by means of a point-of-care device that provided an actual INR value for patients receiving warfarin and a sham INR value for patients receiving Xaban. INR measurements were performed at least monthly and the time in therapeutic range (TTR) been kept over $60 \%$ during as follow up period.

Anticoagulation was continued for at least 3 months. Patients underwent monthly assessment in the out-patients-clinic regarding proper taking medicine, any adverse events and symptom changes. All patients followed up were continued to 6 months and to be contacted at 12 months. To assess thrombosed vein segments recanalization, we have performed duplex scan before start medication and after complete anticoagulation on last follow up visit after complete medication.

Ultrasonography (LOGIQ P9, GE Healthcare, Marlborough, Mass) was done with 5-10 MHz linear probe or 2.5-5 $\mathrm{MHz}$ convex transducer depending on the depth of veins to be examined. The examination of the lower limb veins is started with thigh to visualize the common, deep, and su- 
perficial femoral veins. The popliteal vein and the calf veins (anterior tibial, posterior tibial, peroneal) were examined in the sitting position. The convex probe used to scan the iliac veins. B-mode with compression and color doppler flow visualization applied for whole vein. Thrombus finding at every vein segment was recorded. All duplex scan was done by a same vascular technician. To diagnose concurrent pulmonary embolism, chest CT scan was taken in the initial diagnosis. For simplify classification of thrombus quantification, we define complete resolution for total resolving at
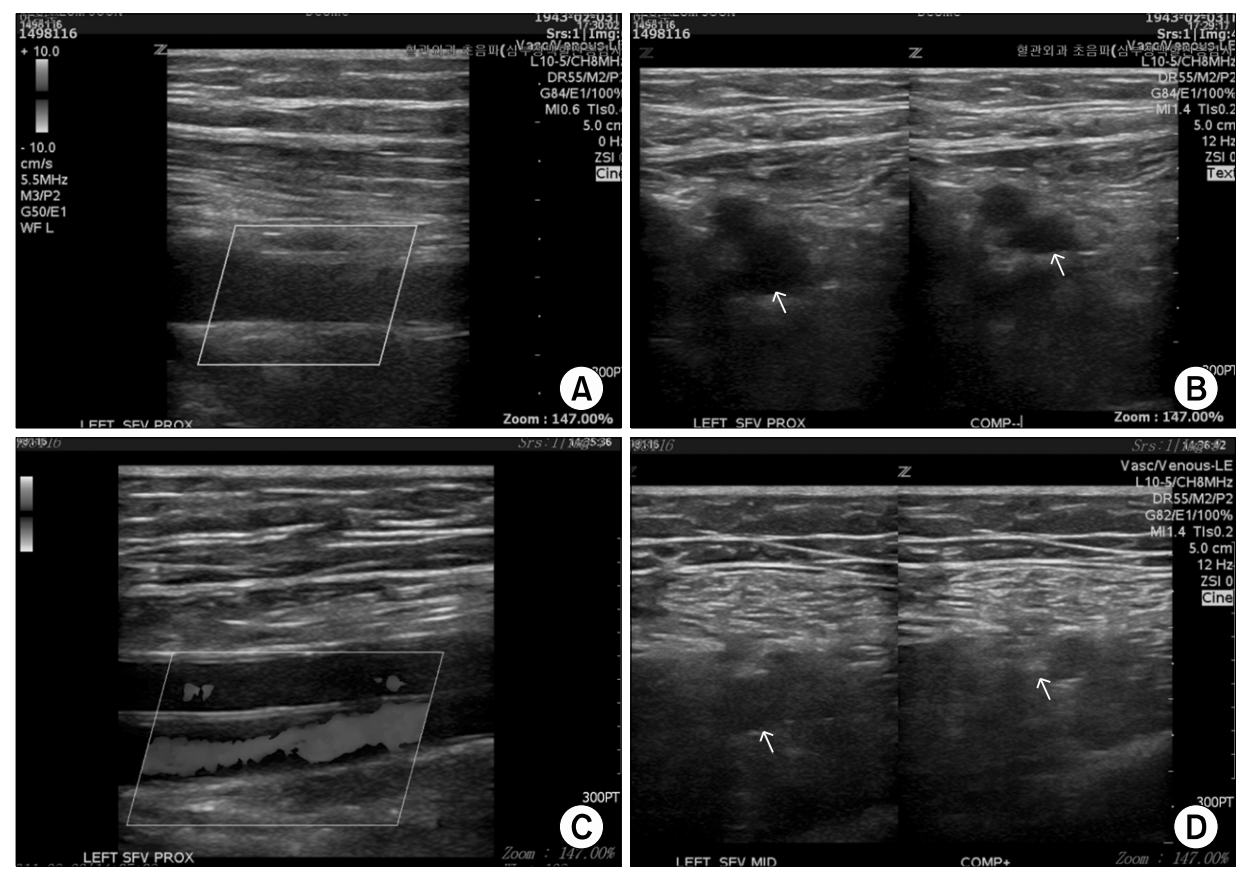

Fig. 1. Thrombus in femoral vein was completely resolved after 6 months Xaban medication: (A) initial finding showed no color signal in color doppler view and (B) thrombosed vein was not compressed; (C) after 6 months, flow was restored with color dopper view and (D) complete obliteration in compression test.
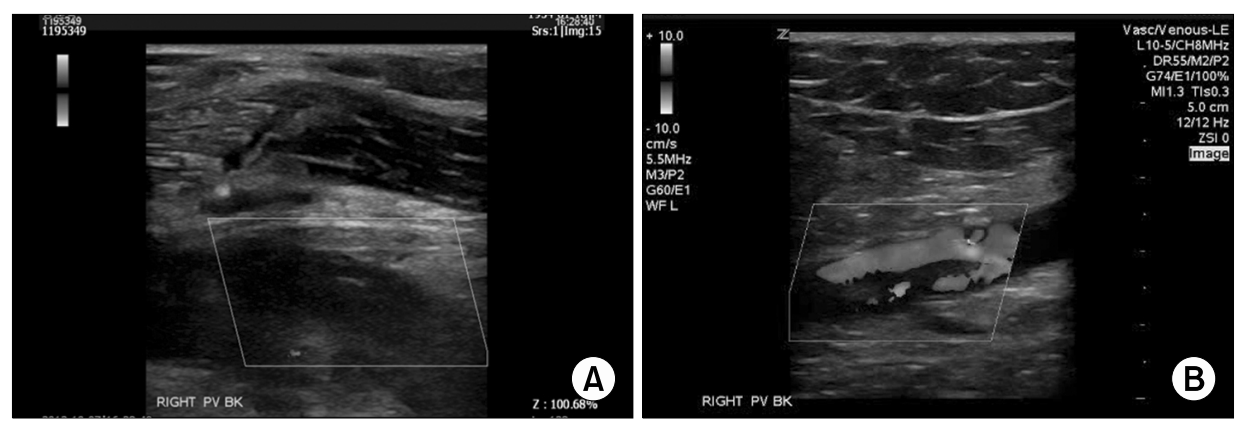

Fig. 2. After 6 months warfarin medication, partial resolution was observed: (A) Initial total occlusion with thrombus in popliteal vein; (B) after long-term medication, partial thrombus resolution with limited flow restoration.

Table 1. Baseline Characteristics of Patients

\begin{tabular}{lccc}
\hline & Xaban group, $\mathrm{n}=38$ & Warfarin group, $\mathrm{n}=19$ & P-value \\
\hline Female sex, no. (\%) & $17(44.7)$ & $9(47.3)$ & 0.851 \\
Mean age, $\mathrm{y}( \pm \mathrm{SD})$ & $60.1( \pm 14.02)$ & $60.1( \pm 13.1)$ & 0.470 \\
Type of DVT, no. $(\%)$ & & & \\
$\quad$ Unprovoked & $31(81.5)$ & $15(78.9)$ & 0.812 \\
$\quad$ Provoked & $7(18.5)$ & $4(21.0)$ & 1.000 \\
Left leg involvement, no. (\%) & $26(68.4)$ & $13(68.4)$ & 1.000 \\
Concomitant pulmonary embolism, no. (\%) & $20(52.6)$ & $10(52.6)$ & \\
\hline
\end{tabular}


each vein segment and partial for any degree of thrombus reduction than no resolution (Figs. 1, 2). Statistics was done by chi-square test and binary logistic regression.

\section{RESULTS}

The study protocol was approved by the Institutional Review Board of Daegu Catholic University Hospital (IRB no. CR-14-081). All patients had presented symptomatic lower extremity swelling. The baseline characteristics of the patients were similar in the two study groups (Table 1). Subjects included 26 female patients and had mean age of 60.1 years ( $S D \pm 13.99$ years and range, 23 to 80 years). All fifty-seven patients have been completed study follow up protocol. No patients were lost to follow up during 12 months period. The left limb was involved in 39 patients (68.4\%) and no patients had bilateral involvement. Regarding the type of DVT, provoked type was 19.2\% (11/57). Thirty-eight patients were randomized to Xaban group and nineteen patients for Warfarin group. Concurrent pulmonary embolism was diagnosed in Xaban group $20(52 \%)$ patients and $10(50 \%)$ patients in warfarin

Table 2. Thrombosis Involving Venous Segments

\begin{tabular}{lcc}
\hline $\begin{array}{c}\text { Proximal thrombosed } \\
\text { vein segment }\end{array}$ & $\begin{array}{c}\text { Xaban group, } \\
\mathrm{n}=38\end{array}$ & $\begin{array}{c}\text { Warfarin group, } \\
\mathrm{n}=19\end{array}$ \\
\hline Inferior vena cava & 2 & 0 \\
Iliac vein & 15 & 8 \\
Femoral vein & 16 & 9 \\
Popliteal vein & 5 & 2 \\
\hline
\end{tabular}

group.

Initial thrombus extent in venous system reported in Table 2. Thrombosis involved proximal vein which defined as inferior vena cava or iliac vein in 25 patients (43.8\%) and located femoro-popliteal vein segments in 32 patients (56.1\%). The proximal levels of thrombus in Xaban group were Inferior vena cava in 2, iliac 15 , femoral 16 and above knee popliteal vein in 5 patients. In Warfarin group, iliac 8 , femoral 9, and popliteal vein were in 2 patients.

Among patients receiving warfarin, the INR was in the therapeutic range for $63.5 \%$ of the time. No VTE related death was encountered in both groups during the study period. The complete resolution rate in proximal segment was $47.3 \%$ (18/38) and partial resolution rate was 51\% (19/38) in Xaban group (Table 3). In warfarin group complete resolution was $57 \%(11 / 19)$ and partial resolution was $57 \%$ (11/19). Whole DVT resolution was 26.3\% (10/38) in Xaban, $42.1 \%$ (8/19) in warfarin. There was no statistically difference regarding proximal resolution $(\mathrm{P}=0.657)$ and

Table 4. Bleeding Complication between Two Groups

\begin{tabular}{lccc}
\hline & $\begin{array}{c}\text { All bleeding } \\
\text { events, no (\%) }\end{array}$ & $\begin{array}{c}\text { OR } \\
\text { (odds ratio) }\end{array}$ & $\begin{array}{c}95 \% \text { CI } \\
\text { for OR }\end{array}$ \\
\hline Group & & & \\
Warfarin $(\mathrm{n}=19)$ & $6(31.58)^{*}$ & 2.462 & $0.669-9.053$ \\
Xaban $(\mathrm{n}=38)$ & $6(15.79)^{\dagger}$ & 1 & - \\
P-value & 0.168 & 0.175 & \\
\hline
\end{tabular}

*Two major bleeding events (huge muscle hematoma, severe sclera hemorrhage) which defined as a condition needs for hospitalization included.

${ }^{\dagger}$ One major bleeding, melena event included and there was no active bleeding focus in endoscopy.

Table 3. 12 Months Follow Up Thrombus Resolution Outcome with Duplex Scan

\begin{tabular}{lcccc}
\hline \multirow{2}{*}{ Variable } & \multicolumn{3}{c}{ Group } & P-value \\
\cline { 2 - 3 } & Warfarin $(\mathrm{n}=19)$ & Xaban $(\mathrm{n}=38)$ & Total $(\mathrm{n}=57)$ & \\
\hline Proximal thrombus resolution, no (\%) & & & & \\
$\quad$ Complete & $11(57.89)$ & $18(47.37)$ & $29(50.88)$ & 0.605 \\
$\quad$ Partial & $7(36.84)$ & $19(50)$ & $26(45.61)$ & \\
$\quad$ Never & $1(5.26)$ & $1(2.63)$ & $2(3.51)$ & 0.227 \\
Whole venous segment resolution, no (\%) & $11(57.89)$ & $28(73.68)$ & $39(68.42)$ & \\
$\quad$ Residual thrombus & $8(42.11)$ & $10(26.32)$ & $18(31.58)$ & \\
$\quad$ Total resolution & &
\end{tabular}


complete resolution rate $(P=0.227)$ between two groups. For initial coagulation anomaly function test, 85.9\% (49/57) patients showed at least one anomalous result. The most frequent abnormality was protein $\mathrm{S}$ activity reduction \% $80.7 \%(46 / 57)$.

There was no VTE recurrence during 12 months follow up in both groups. Clinically relevant bleeding complication (major or minor) occurred in 6 of 38 patients $(15.7 \%$ ) in the Xaban group and in 6 of 19 patients (31.5\%) in warfarin group and odds ratio was $2.462(95 \% \mathrm{CI}$ for OR, $\mathrm{P}=0.175)$ (Table 4).

\section{DISCUSSION}

Prevention of VTE recurrence and PTS is the most important therapeutic goals in acute DVT patients. During natural course of long-term anticoagulation, variable degree of thrombus may remain in thrombosed vein segments. RT after completion of anticoagulation was reported that it closely related with these two adverse outcomes. A number of studies have explored the role of RT and the associated risk of VTE recurrence. A recent meta-analysis demonstrated an elevated odds or VTE recurrence in DVT patients with residual vein thrombosis 2.5, (95\% CI 1.1-2.0).(12) However thrombus evolution is dynamic and complex procedure that involves organization and neovascularization as well as fibrinolysis so it remained poorly defined. Application ultrasound for diagnosis of thrombus remnant status also widely reported in the literature. Killewich et al.(13) reported that 55\% of acute DVT patients showed complete recanalization within 6 to 9 months. In DACUS study, the adjusted hazard ratio of residual thrombus versus those without was 24.9 (95\% CI, 3.4-183.6; $\mathrm{P}=0.002$ ) and absence of thrombus identifies patients at low risk for recurrent VTE.(14) Warfarin promotes fibrinolysis by reducing thrombin induced activation of thrombin activatable fibrinolysis inhibitor.(15) During warfarin medication, proper maintain of INR value is critical for thrombus resolution. Caprini et al. (8) reported that at the end of 1-year follow up, the rate of complete DVT resolution was $68 \%$ and the median INR values significantly lower with incomplete DVT resolution. Also in this less thrombus resolved group, $62 \%$ had chronic venous insufficiency symptoms develop, whereas only $11 \%$ of the patients with non-occlusive thrombi $(\mathrm{P}=0.003)$. In our study, complete whole venous thrombus resolution rate in warfarin was $42.1 \%$ (8/19) and 26.3\% (10/38) in Xaban group. Our results showed similar resolution rate with others prospective study in which after 3 months anticoagulation $42.86 \%$ of provoked proximal DVT patients showed complete resolution.(16) In our study Xaban group outcome revealed higher RT rate without statistical significance. Since thrombus resolution observed in proximal vein, we also studied the evolution of the most proximal thrombus in DVT and it showed no significant difference in two anticoagulation groups. It is known that compare to distal thrombus, proximal DVT has increased risk of recurrence (odds ratio $=2.4 ; 95 \%$ confidence interval [CI]: $0.95,5.9$ ) as in Douketis et al. report.(17) In the future, larger scale of prospective study is warranted to find out more robust results.

Although Xaban also block the thrombin action by sequential interaction beginning with factor Xa, the scope of interaction with coagulation factor is different with warfarin. So far there is no specific study defining what would be the result of this complex interaction in thrombus evolution with NOAC. Although many Xaban trials reported VTE recurrence, no specific PTS occurrence was reported. However warfarin has intrinsic limitations such as unpredictable pharmacokinetics, monitoring requirements, drug-food or drug-drug interactions. Also because of bleeding risk, physicians tend to be monitored in subtherapeutic INR level. In population based real-world research with 19,113 VTE patients, only 36\% had TTR value over 70\%.(18) There is a high amount of unpredictable variability in an individual TTR. These findings confirm the difficulty of achieving high quality anticoagulation with warfarin in real-world clinical practice. In our study warfarin assigned patient's TTR was $63.5 \%$ and it is essential for comparing proper therapeutic effect evaluation. Compared 
with warfarin, NOACs has more predictable anticoagulant responses and it have been shown to be effective in the prevention and treatment of VTE. Also NOACs has little food interactions, or drug interaction with predictable pharmaco-kinetics it could be administered with fixed dose without laboratory monitoring. So far three (Rivaroxaban, Apixaban, Edoxaban) direct factor Xa inhibitor drug approved from Food and Drug Administration for VTE treatment. In all NOAC trials it is found that these drugs found to be non-inferior to warfarin with regards to the primary efficacy outcomes and fewer major bleeding events.( 9 -11) However all these trials had similar outcome assessments for primary efficacy and it was recurrent venous thromboembolism. Even with high quality study design with strict follow up protocol, final thrombus resolution results compare with conventional anticoagulation were not clearly mentioned especially imaging final outcomes.

Our randomized study was focused the thrombus recanalization rate comparison. Study patients characteristics were not different with other studies. Unprovoked vs. provoked DVT ratio (78.9\% vs. $21 \%)$ in warfarin group were similar with DACUS trial (75\% vs. $25 \%)$.(14) Clinical relevant bleeding event was higher in warfarin group $31.5 \%$ vs. $15.7 \%$ without significance. Although the rates was higher to Xaban phase III trials (Apixaban 8.0\% vs. 3.8\%, Edoxaban $10.3 \%$ vs. $8.5 \%$, Rivaroxaban $8.1 \%$ vs. $8.1 \%$ ), most of bleeding events were minor bleeding such as bruising, menorrhagia, oral mucosa ecchymosis and subconjunctival hemorrhage. There were two major bleeding events occurred in warfarin group which defined as the condition needs to be hospitalized. One patient admitted for temporary hematemesis and the other patient was for muscle hematoma in thigh.

Both patients treated with conservative treatment without any intervention. RT occlusion also has close relation with PTS which is a late complication of DVT that greatly affects the morbidity and quality of life of patients and leads to high social costs. Limbs with the PTS had more than three times the odds of having combined reflux and obstruction than did limbs without the PTS (odds ratio =
3.5, 0.95 confidence intervals $=1.4,8.6$ ) with duplex scan test.(19) In our study TR rate was not significantly different Xaban with warfarin. Based on this finding, we hypothesize that the incidence of PTS would not be influenced by the choice of these two different drugs. However when we consider that rather lower level of INR maintenance for warfarin prescription in practical VTE treatment, it is presumed that PTS could be frequent with conventional treatment. Further real world comparison study need to be find out this subject. Duplex sonography is the method of choice for diagnosis of DVT since it offers both anatomic and hemodynamic information with gray scale imaging and pulsed Doppler waveform analysis. There were some studies to objectify the extent of thrombus with ultrasonogram.(20) However even with high validity of scoring system, the complexity decrease the real-world application especially extensive leg thrombosis. Instead of using thrombus scoring index, we simplify the TR with total resolution, partial and no resolution. It is practical for follow up thrombus absorption in whole segment for comparing the changes of thrombus volume. We also focused the most proximal thrombus resolution during the follow up image to define longitudinal thrombus regression.

In conclusion, Xaban administration for acute VTE showed no difference regarding TR with warfarin. Based on this result there is a possibility that PTS related with RT would not be different between new and conventional anticoagulant. Because Xaban does not require monitoring and has a short half-life, Xaban could also be an easy-to-use anticoagulation treatment option. Further larger scales study expect to warrant our results.

\section{CONFLICT OF INTEREST}

The authors have nothing to disclose.

\section{ACKNOWLEDGEMENTS}

This work was supported by research grants from Daegu Catholic University in 2017. 


\section{REFERENCES}

1. Prandoni P, Noventa F, Ghirarduzzi A, Pengo V, Bernardi E, Pesavento R, et al. The risk of recurrent venous thromboembolism after discontinuing anticoagulation in patients with acute proximal deep vein thrombosis or pulmonary embolism. A prospective cohort study in 1,626 patients. Haematologica 2007;92:199-205.

2. Iorio A, Kearon C, Filippucci E, Marcucci M, Macura A, Pengo $\mathrm{V}$, et al. Risk of recurrence after a first episode of symptomatic venous thromboembolism provoked by a transient risk factor: a systematic review. Arch Intern Med 2010;170:1710-6.

3. Prandoni P, Lensing AW, Prins MH, Bernardi E, Marchiori A, Bagatella $P$, et al. Residual venous thrombosis as a predictive factor of recurrent venous thromboembolism. Ann Intern Med 2002:137:955-60.

4. Young L, Ockelford P, Milne D, Rolfe-Vyson V, Mckelvie S, Harper P. Post-treatment residual thrombus increases the risk of recurrent deep vein thrombosis and mortality. J Thromb Haemost 2006;4:1919-24.

5. Kahn SR, Shrier I, Julian JA, Ducruet T, Arsenault L, Miron MJ, et al. Determinants and time course of the posthrombotic syndrome after acute deep venous thrombosis. Ann Intern Med 2008;149:698-707.

6. Killewich LA, Bedford GR, Beach KW, Strandness DE Jr. Spontaneous lysis of deep venous thrombi: rate and outcome. J Vasc Surg 1989;9:89-97.

7. Murphy TP, Cronan JJ. Evolution of deep venous thrombosis: a prospective evaluation with US. Radiology 1990177:543-8.

8. Caprini JA, Arcelus JI, Reyna JJ, Motykie GD, Mohktee D, Zebala LP, et al. Deep vein thrombosis outcome and the level of oral anticoagulation therapy. J Vasc Surg 1999;30:805-11.

9. Agnelli G, Buller HR, Cohen A, Curto M, Gallus AS, Johnson M, et al. Oral apixaban for the treatment of acute venous thromboembolism. N Engl J Med 2013;369:799-808.

10. Bauersachs R, Berkowitz SD, Brenner B, Buller HR, Decousus $\mathrm{H}$, Gallus AS, et al. Oral rivaroxaban for symptomatic venous thromboembolism. N Engl J Med 2010;363:2499-510.

11. Büller HR, Décousus H, Grosso MA, Mercuri M, Middeldorp S, Prins $\mathrm{MH}$, et al. Edoxaban versus warfarin for the treatment of symptomatic venous thromboembolism. N Engl J Med
2013;369:1406-15.

12. Carrier M, Rodger MA, Wells PS, Righini M, LE Gal G. Residual vein obstruction to predict the risk of recurrent venous thromboembolism in patients with deep vein thrombosis: a systematic review and meta-analysis. J Thromb Haemost 2011; 9:1119-25.

13. Killewich LA, Macko RF, Cox K, Franklin DR, Benjamin ME, Lilly MP, et al. Regression of proximal deep venous thrombosis is associated with fibrinolytic enhancement. J Vasc Surg 1997; 26:861-8.

14. Siragusa S, Malato A, Anastasio R, Cigna V, Milio G, Amato C, et al. Residual vein thrombosis to establish duration of anticoagulation after a first episode of deep vein thrombosis: the Duration of Anticoagulation based on Compression UltraSonography (DACUS) study. Blood 2008;112:511-5.

15. Incampo F, Carrieri C, Galasso R, Scaraggi FA, Di Serio F, Woodhams B, et al. Effect of warfarin treatment on thrombin activatable fibrinolysis inhibitor (TAFI) activation and TAFI-mediated inhibition of fibrinolysis. J Thromb Haemost 2013;11:315-24.

16. Brownson K, Satoskar S, Reynolds J, Sumpio B, Sarac T, Scoutt $\mathrm{L}$, et al. Thrombus resolution as guide to anticoagulation therapy for provoked deep vein thrombosis: TRUDVT pilot study. J Vasc Surg Venous Lymphat Disord 2016;4:149.

17. Douketis JD, Crowther MA, Foster GA, Ginsberg JS. Does the location of thrombosis determine the risk of disease recurrence in patients with proximal deep vein thrombosis? Am J Med 2001;110:515-9.

18. Macedo AF, Bell J, McCarron C, Conroy R, Richardson J, Scowcroft A, et al. Determinants of oral anticoagulation control in new warfarin patients: analysis using data from Clinical Practice Research Datalink. Thromb Res 2015;136:250-60.

19. Johnson BF, Manzo RA, Bergelin RO, Strandness DE Jr. Relationship between changes in the deep venous system and the development of the posthrombotic syndrome after an acute episode of lower limb deep vein thrombosis: a one- to six-year follow-up. J Vasc Surg 1995;21:307-12; discussion 313.

20. Ouriel K, Greenberg RK, Green RM, Massullo JM, Goines DR. A volumetric index for the quantification of deep venous thrombosis. J Vasc Surg 1999;30:1060-6. 